\title{
Fracture Toughening and Toughness Asymmetry Induced by Flexoelectricity
}

\author{
Amir Abdollahi,,${ }^{1,2}$ Christian Peco, ${ }^{1}$ Daniel Millán, ${ }^{1}$ Marino Arroyo, ${ }^{1}$ Gustau Catalan,${ }^{2,3}$ and Irene Arias ${ }^{4, *}$ \\ ${ }^{1}$ Laboratori de Càlcul Numèric (LaCàN), Universitat Politècnica de Catalunya (UPC), \\ Campus Nord UPC-C2, E-08034 Barcelona, Spain. \\ ${ }^{2}$ ICN2-Institut Catala de Nanociència i Nanotecnologia, Bellaterra 08193, Barcelona, Spain \\ ${ }^{3}$ ICREA-Institut Catala de Recerca I Estudis Avançats, Barcelona, Spain \\ ${ }^{4}$ Laboratori de Càlcul Numèric (LaCàN), Universitat Politècnica de Catalunya-Barcelonatech (UPC), \\ Campus Nord UPC-C2, E-08034 Barcelona, Spain.
}

(Dated: May 8, 2015)

\begin{abstract}
Cracks generate the largest strain gradients that any material can withstand. Flexoelectricity (coupling between strain gradient and polarization) must therefore play an important role in fracture physics. Here we use a self-consistent continuum model to evidence two consequences of flexoelectricity in fracture: the resistance to fracture increases as structural size decreases, and it becomes asymmetric with respect to the sign of polarization. The latter phenomenon manifests itself in a range of intermediate sizes where piezo- and flexoelectricity compete. In BaTiO3 at room temperature, this range spans from 0.1 to $50 \mathrm{~nm}$, a typical thickness range for epitaxial ferroelectric thin films.
\end{abstract}

The best known and most technologically exploited electro-mechanical coupling mechanism in solids is piezoelectricity, by which electric polarization is generated by strain, or conversely strain is generated by an applied electric field. Flexoelectricity is a different electromechanical coupling mechanism, whereby polarization couples to spacial variations of the strain field (strain gradients). Flexoelectricity is a more widespread property than piezoelectricity - it is, in fact, a universal property of all insulators [1-3], and it is particularly strong in materials with high dielectric constants such as ferroelectrics [4-9].

Because of the size-dependence of strain gradients, flexoelectricity is most conspicuous at the nanoscale. At these scales, large strain gradients can be achieved without rupture, leading to large flexoelectric effects in samples such as thin films [10] and near nano-inclusions $[11,12]$. Recent nano-indentation experiments on ferroelectrics have shown a strong size-dependent stiffening $[13,14]$ and electric-field-dependent elastic modulus [15], attributed to flexoelectricity caused by the highly inhomogeneous strain field produced by the nanoindenter. Flexoelectric sensors based on ferroelectrics have been proposed to detect localized damage where strain gradients are prominent [16]. These results highlight the effect of flexoelectricity on mechanical properties, and suggest that flexoelectricity may play an important and hitherto overlooked role in fracture physics.

The ultimate strain gradient that any material can withstand is that which exists around a crack tip: the material just before the crack apex is, by definition, on the verge of rupture, and hence under the maximum deformation it can stand, while the distance over which this strain is released tends towards the atomic scale at the apex. Cracks are therefore a natural ground where to search for flexoelectric effects.

In this paper, we analyze the fracture of ferroelectrics using a self-consistent model of flexoelectricity [17]. Macroscopic experiments [18, 19] have already shown that the resistance to cracking depends on the direction of polarization, in agreement with theoretical models coupling fracture and ferroelectricity [20, 21]. Here, we find that, in the presence of flexoelectricity, the resistance to fracture significantly increases (fracture toughening) in a size-dependent manner, and moreover that this toughening is asymmetric with respect to the sign of the polarization.

To examine the role of flexoelectricity on fracture physics of polar materials, we consider a linear continuum theory of piezoelectricity with poling [25], augmented with flexoelectricity [26]. We discuss later the quantitative limitations of this simple but transparent model. The electrical enthalpy density of a poled piezoelectric solid possessing piezoelectricity and flexoelectricity can be written as

$$
\begin{aligned}
& \mathcal{H}\left(\varepsilon_{i j}, E_{i}, \nabla_{l} \varepsilon_{j k}\right)=\frac{1}{2} \mathbb{C}_{i j k l} \varepsilon_{i j} \varepsilon_{k l}-e_{i k l} E_{i} \varepsilon_{k l} \\
& -\mu_{i j k l} E_{i} \nabla_{l} \varepsilon_{j k}+\frac{1}{2} g_{i j k l m n} \nabla_{k} \varepsilon_{i j} \nabla_{n} \varepsilon_{l m}-\frac{1}{2} k_{i j} E_{i} E_{j},
\end{aligned}
$$

where $\mathbf{E}$ is the electric field, defined as $E_{i}=-\nabla_{i} \phi, \phi$ being the electric potential. The first term is the elastic potential, where $\mathbb{C}$ is the fourth-rank tensor of elastic moduli. The piezoelectric coupling between strain and electric field is through the second term via the thirdrank tensor of piezoelectricity e. The last term is the electrostatic potential, where $\mathbf{k}$ is the second-rank dielectric tensor. The flexoelectric coupling between the gradient of strain $\nabla \varepsilon$ and the electric field is through the third term, where the fourth-rank flexoelectric tensor $\boldsymbol{\mu}$ describes both direct and converse flexoelectric effects $[27,28]$. The fourth term is the strain gradient elastic potential, $\mathrm{g}$ being the sixth-rank strain gradient elasticity tensor. This term is usually discarded, but it is important in the presence of strong elastic gradients, and it guar- 
antees the thermodynamic stability of the model in the presence of flexoelectricity [11, 29-31]. In this formulation, the remanent state of the piezoelectric material has been taken as the reference configuration, and the poling of the piezoelectric material is implicitly encoded in the symmetry of the piezoelectric tensor e [25]. The electric displacement $\mathbf{D}$ can be derived from Eq. (1) by differentiating with respect to $-\mathbf{E}$. Since $\mathbf{D}=\varepsilon_{0} \mathbf{E}+\mathbf{P}$, where $\mathbf{P}$ is the electric polarization and $\varepsilon_{0}$ is the permittivity of free space, and introducing the dielectric susceptibility $\chi_{i j}=k_{i j}-\varepsilon_{0} \delta_{i j}$, we obtain the constitutive relation for polarization as

$$
P_{i}=\chi_{i j} E_{j}+e_{i k l} \varepsilon_{k l}+\mu_{i j k l} \nabla_{l} \varepsilon_{j k},
$$

which includes dielectric, piezoelectric, and flexoelectric contributions [1,32].

In the absence of surface charges, the total electromechanical enthalpy is

$$
H=\int_{\Omega} \mathcal{H} \mathrm{d} \Omega-\int_{\Gamma_{t}} \bar{t}_{i} u_{i} \mathrm{~d} S
$$

where $\overline{\mathbf{t}}$ are the mechanical tractions applied on the boundary. The self-consistent governing equations in weak form for the electro-mechanical boundary value problem follow from making the enthalpy stationary with respect to the displacement and electric potential fields [17]. Numerically, we deal with the fourth-order nature of the partial differential equations by approximating displacements and electric potential using a meshfree method with smooth basis functions [33].

To evaluate the role of flexoelectricity on the fracture of ferroelectrics, we compute energy release rates in a poled specimen. In Griffith's theory, fracture occurs when the energy release rate $G$ reaches the energetic fracture toughness of the material $G_{c}$. Therefore, a decrease in $G$ due to the electromechanical coupling is interpreted as a toughening effect on the material [34, 35]. The energy-release rate $G$ is defined as the reduction in the potential energy of the cracked body per unit increase in the crack length $a$ (in two dimensions). Considering two cracked plane specimens with the same material, configuration, loading and boundary conditions, and cracks of slightly different length $\Delta a$, the energy release rate can be computed as $G=[H(a)-H(a+\Delta a)] / \Delta a$, where $H$ is given in Eq. (3).

We consider two pre-cracked beams, with pre-crack lengths of $a=h / 4$ and $a=h / 4+h / 1000(\Delta a=$ $h / 1000)$, in a four-point bending configuration as shown in Fig. 1(a), with the material properties of barium titanate $\left(\mathrm{BaTiO}_{3}\right)[36]$ and poled along the $x_{2}$-axis. We consider both the longitudinal and transversal flexoelectric coefficients $\mu_{11}$ and $\mu_{12}$. Given that there is not yet a universal consensus regarding the size, or even the sign, of these coefficients for any material [2], we consider three cases C1: $\mu_{11}=\mu_{12}, \mathrm{C} 2: \mu_{12}=-10 \mu_{11}$,
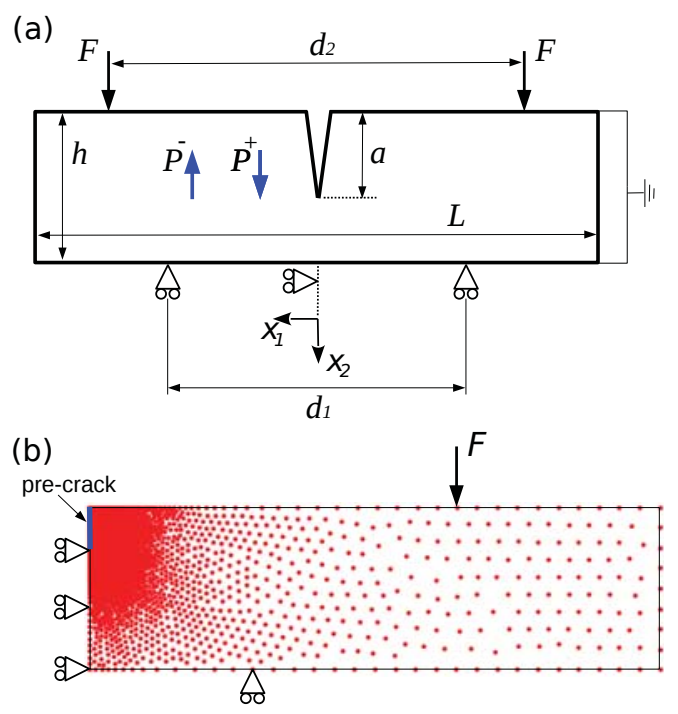

FIG. 1. (a) Four-point bending set up with dimensions $L=$ $7 h=3.5 d_{1}=1.5 d_{2}$. The specimen is poled along the positive $\left(P^{+}\right)$and negative $\left(P^{-}\right) x_{2}$ directions, i.e. parallel and antiparallel to the crack, respectively. The top and bottom sides are connected to the ground. (b) Computational node set.

and C3: $\mu_{11}=2 \mu_{12}$, where the latter two limiting cases are chosen according to reported values for ferroelectrics $[7,37,38]$. The magnitude of these coefficients is chosen as $\mu=\chi f$, where $f$ is the flexocoupling coefficient and the dielectric susceptibility of $\mathrm{BaTiO}_{3}$ at room temperature is $\chi_{11}=36 \mathrm{nC} \cdot(\mathrm{Vm})^{-1}$ along the a-axis and $\chi_{33}=1.7 \mathrm{nC} \cdot(\mathrm{Vm})^{-1}$ along the c-axis [39]. The value of $f$ has been estimated to be of the order of $1-10 \mathrm{~V}$ for simple ionic solids [2]. We choose the maximum value of $f=10 \mathrm{~V}$, as confirmed by measurements on single crystals [40]. Although surface piezoelectricity is theoretically expected to contribute to the total flexoelectric coefficient [41], we do not incorporate it as a separate term since (i) the actual thickness and piezoelectric coefficient of the surface have not yet been characterized for any material and (ii) the effect of surface piezoelectricity is contained within the effective flexoelectric coefficient $[41,42]$. The parameter $g=1.4 \times 10^{-9} \mathrm{~N}$ is chosen to satisfy the stability condition for the flexoelectric equations [31]. A point load $F=9 \times 10^{4} \sqrt{h} \mathrm{Nm}^{-1 / 2}$ is considered to supply sufficient energy for fracture at different length scales, i.e. the calculated energy release rates are in the order of the energetic fracture toughness of $\mathrm{BaTiO}_{3}$, $G_{c}=2\left(\mathrm{Jm}^{-2}\right)[20]$.

The energy-release rate is obtained as a function of the beam thickness $h$ with the poling direction parallel (positive) and anti-parallel (negative) to the pre-crack, see Fig. 1(a). To screen the surface depolarization field, we assume that the top and bottom sides of the beam are connected to the ground, i.e., the electric potential is fixed to zero, while other sides are charge-free. The 
aspect ratio of the beam is fixed to $L / h=7, L$ being the length of the beam. For symmetry reasons, only the right half of the beam is analyzed. A non-uniform node distribution is considered for the model discretization, with higher concentration of nodes around the crack tip, as shown in Fig. 1(b).

Figure 2(a) presents the energy release rate $G$ as a function of the beam thickness $h$ for the poled piezoelectric beam with and without considering flexoelectricity. As expected, without the flexoelectric effect, the energy release rate is independent both on the size of the beam and the poling direction, see Fig. 2(a). By introducing flexoelectricity, we observe two effects: (1) the energy release rate decreases with the beam thickness, i.e. the thinner the beam, the tougher, and (2) this fracture toughness enhancement is sensitive to the poling direction, which we term fracture toughness asymmetry. For instance, a $78 \%$ reduction in energy release rate is observed for the parallel (positive) poling direction in the $\mathrm{C} 2$ case for a thickness of $2.4 \mathrm{~nm}$, corresponding to the thinnest $\mathrm{BaTiO}_{3}$ film retaining ferroelectricity [43]. While for $\mathrm{BaTiO}_{3}$ smaller sizes are not physically meaningful, we report results corresponding to thicknesses below $2.4 \mathrm{~nm}$ to examine the general features of fracture physics in the presence of flexoelectricity, which may be relevant for other materials that preserve piezoelectricity down to lower film thickness. We note that in these simulations, the strain gradient elasticity term involving $\mathbf{g}$ in Eq. (1) is theoretically required to maintain the positive definiteness of the system, but has been previously shown to be insignificant in other situations [30]. Here, for a thickness below about $10 \mathrm{~nm}$, this term is necessary to maintain thermodynamic stability.

To understand the origin of the fracture toughness enhancement, we plot maps of polarization magnitude for different beam thicknesses, shown in Fig. 2(b) for the positively poled piezoelectric beam with flexoelectricity in the $\mathrm{C} 2$ case. Since the electric field is induced by the flexo- and piezoelectric effects, we can graphically represent the polarization pattern by focusing on the dielectric polarization $\mathbf{P}_{e}=\chi \mathbf{E}$. As the beam thickness decreases, the relative size of the flexoelectrically polarized region around the crack tip increases. A similar and smaller effect is also observed for the negatively poled piezoelectric beam and for the other two cases C1 and C3 (not shown). In the absence of flexoelectricity, we obtain a polarization magnitude map similar to that in Fig. 2(b)I irrespective of the beam thickness.

The response of the beam with the flexoelectric effect is also sensitive to the poling direction. Figure 2(a) shows that the energy release rate of the beam $(\mathrm{C} 2)$ with the positive poling direction is lower than that with negative poling direction, i.e. the beam poled parallel to the crack is tougher. This direction is reversed in the $\mathrm{C} 1$ and $\mathrm{C} 3$ cases. Figure 3 presents the fracture toughness asymmetry obtained as the absolute percentage difference be-
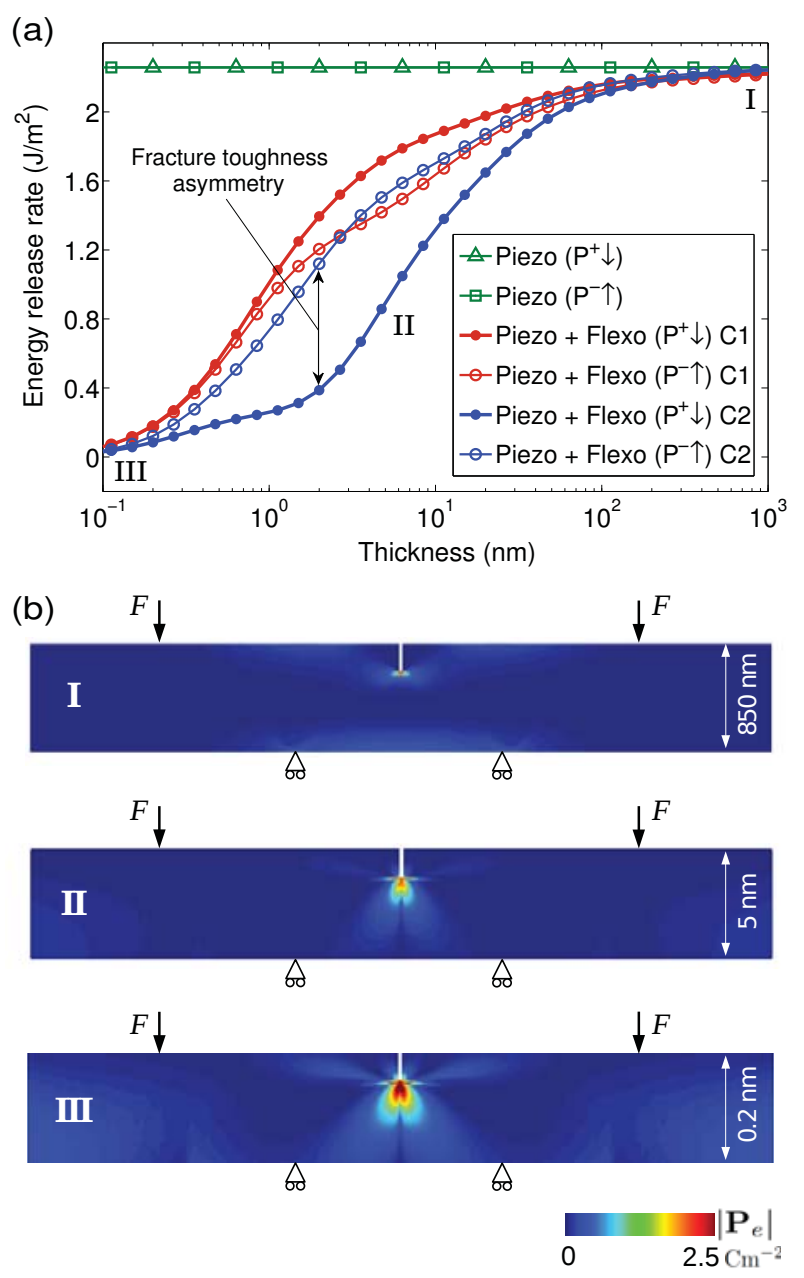

FIG. 2. (a) Energy release rate as a function of the thickness of the poled piezoelectric beam with and without flexoelectricity. The results are obtained considering the material is poled parallel $(+)$ and anti-parallel (-) to the crack. Two cases of the flexoelectric coefficients (C1: $\mu_{11}=\mu_{12}$ and $\left.\mathrm{C} 2: \mu_{12}=-10 \mu_{11}\right)$ are considered for the piezoelectric beam with flexoelectricity. (b) Distribution of the polarization magnitude $\left|\mathbf{P}_{e}\right|$ for three different thicknesses (marked as I - III above) considering the positively poled piezoelectric beam with flexoelectricity in the $\mathrm{C} 2$ case.

tween the energy release rates of the negatively and positively poled beams with flexoelectricity, as a function of the beam thickness. The insets show the polarization field in a small area around the crack tip for three different beam thicknesses for both the negatively and positively poled material in the $\mathrm{C} 2$ case. A significant asymmetry is observed in a wide range of beam thickness (spanning over one order of magnitude).

For thick beams, the response is dominated by piezoelectricity, Fig. $3(\mathrm{I} \pm)$, while for thin beams, the response is mainly due to flexoelectricity, Fig. 3(III \pm ). The polarization fields induced by piezoelectricity in Figs. 3(I) and $(\mathrm{I}+)$ are antisymmetric with respect to changing the poling sign, i.e. they are identical in magnitude but 


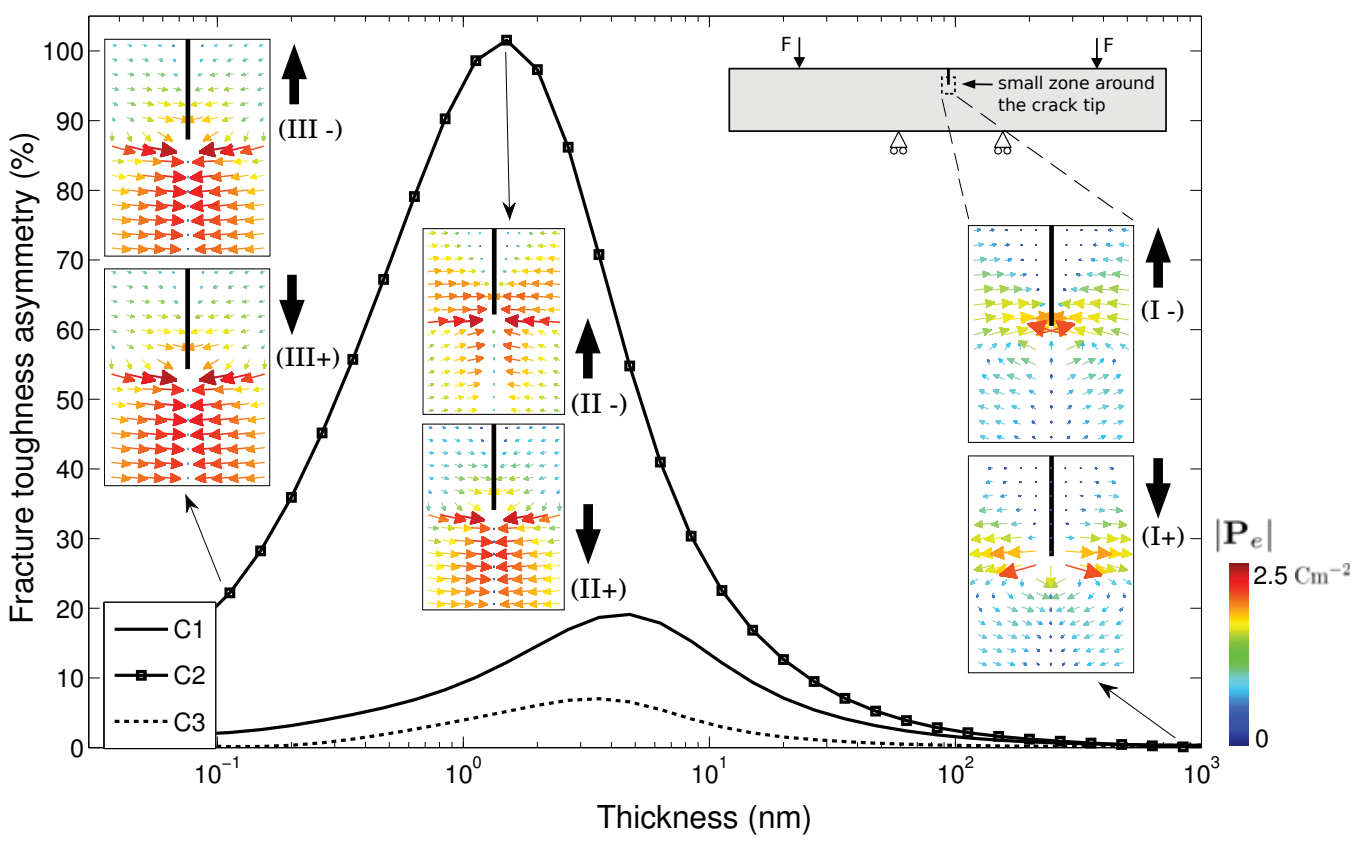

FIG. 3. Fracture toughness asymmetry as a function of the beam thickness. The asymmetry is obtained as the absolute percentage difference between the energy release rates of the negatively and positively poled piezoelectric beams with flexoelectricity, presented in Fig. 2 for two cases of the flexoelectric coefficients $\left(\mathrm{C} 1: \mu_{11}=\mu_{12}\right.$ and $\left.\mathrm{C} 2: \mu_{12}=-10 \mu_{11}\right)$, and the additional case C3: $\mu_{11}=2 \mu_{12}$. The insets show the distribution of the polarization $\mathbf{P}_{e}$ in a small zone around the crack tip at three different length-scales for the negative (top) and positive (bottom) poling directions in the C2 case. The bold black line shows the position of the pre-crack and the bold black arrow indicates the poling direction of the beam.

have reverse direction. As shown in Fig. 2(a), this sign reversal does not affect the material toughness. Turning now to the small-scale limit, the flexoelectric-induced fields are symmetric with respect to changing the poling sign, i.e. they do not depend on the poling direction, see Figs. 3(III-) and (III+), since they are induced by strain gradients. Therefore, if either piezo- or flexoelectricity dominates the response, reversing the poling direction of the material does not affect the fracture toughness. In contrast, for intermediate sizes, flexo- and piezoelectricity are comparable effects, and may therefore help or counteract each other; for a positively poled material, the piezo- and flexoelectric polarization fields point in the same direction in front of the crack and in the opposite direction in its wake, while this situation is reversed for a negatively poled material, resulting in a fundamentally different polarization pattern, see Figs. 3(II-) and (II+). This explains the significant fracture toughness asymmetry at intermediate beam sizes.

For $\mathrm{BaTiO} 3$ at room temperature, the range with significant fracture toughness asymmetry is between $0.1 \mathrm{~nm}$ and $50 \mathrm{~nm}$ approximately. This is a typical thickness range for epitaxial ferroelectric thin films. For the critical thickness $2.4 \mathrm{~nm}$, the asymmetry reaches a considerable value of about $90 \%$ in the $\mathrm{C} 2$ case; such enormous toughness asymmetry should be observable via nanoindentation experiments in epitaxial films.
Domain switching makes the fracture response of ferroelectrics more complex [20, 22, 23, 25]. As mentioned earlier, the present model is based on the linear theory of piezoelectricity in the absence of domain switching. However, in ferroelectric materials such as $\mathrm{BaTiO}_{3}$ in the tetragonal phase, ferroelastic switching becomes favorable when the polarization is parallel to the crack. Ferroelastic switching is precluded in $\mathrm{BaTiO}_{3}$ thin films epitaxially clamped onto substrates imposing compressive in-plane strain. Even then, a strong enough flexoelectric field can induce $180^{\circ}$ switching of the material polarization, the so-called flexoelectric switching $[1,2,10]$. If switching occurs, it may lead to energy dissipation near the crack tip, consequently leading to stronger fracture toughness asymmetry.

The calculated size of the flexoelectrically-induced polarization near the tip apex is huge: $2.5 \mathrm{C} / \mathrm{m}^{2}$. This is 10 times bigger than the actual ferroelectric polarization of $\mathrm{BaTiO}_{3}$. This is of course the reason why in the low thickness limit the toughness is insensitive to the ferroelectric orientation. However, the size of the flexoelectric polarization is so big that higher order coupling terms should not be neglected $[24,26]$. It has also been suggested that, because soft materials can withstand larger strain gradients, flexoelectricity is likely to play a significant role in this context $[44,45]$, where fracture is significantly affected by elastic nonlinearity near the crack tip 
[46]. Thus, the inclusion of nonlinear effects is the natural next step in understanding the physics of fracture and flexoelectricity, and we hope this discussion motivates more research also into the measurement of higher order flexoelectric coefficients [24]. It would be very interesting to corroborate our results with atomistic calculations, but unfortunately this is extremely challenging because the physical effects that we are reporting require spanning several orders of magnitude in size.

In summary, the flexoelectric effect leads to a significant enhancement in fracture toughness of $\mathrm{BaTiO}_{3}$ at thicknesses below $50 \mathrm{~nm}$. Importantly, at intermediate scales the fracture toughness is sensitive to the poling direction of the material (parallel or anti-parallel to the crack), which is not the case in the absence of flexoelectricity [20, 21]. Flexoelectricity therefore fundamentally changes the symmetry of fracture physics: while crack propagation was hitherto thought to be invariant with respect to space inversion for all materials, the present work shows that this symmetry is broken in polar materials. This phenomenology can be understood in simple terms from the symmetry and size-dependence of flexoelectricity. We predict that the toughness asymmetry could reach values as high as $100 \%$ for ultra-thin epitaxial films of $\mathrm{BaTiO}_{3}$.

The authors gratefully acknowledge the support of the Ministerio de Ciencia e Innovación (DPI2011-26589). GC's research is supported by an ERC Starting Grant (Project No. 308023), by project FIS2013-48668 from the Ministerio de Ciencia e Innovación, and by the Severo Ochoa Excellence award of ICN2.

* irene.arias@upc.edu

[1] P. V. Yudin and A. K. Tagantsev, Nanotech. 24, 432001 (2013).

[2] P. Zubko, G. Catalan, and A. K. Tagantsev, Annu. Rev. Mater. Res. 43, 387 (2013).

[3] T. D. Nguyen, S. Mao, Y.-W. Yeh, P. K. Purohit, and M. C. McAlpine, Adv. Mater. 25, 946 (2013).

[4] W. Ma and L. E. Cross, Appl. Phys. Lett. 81, 3440 (2002).

[5] W. Ma and L. E. Cross, Appl. Phys. Lett. 86, 072905 (2005).

[6] W. Ma and L. E. Cross, Appl. Phys. Lett. 88, 232902 (2006).

[7] P. Zubko, G. Catalan, A. Buckley, P. R. L. Welche, and J. F. Scott, Phys. Rev. Lett. 99, 167601 (2007).

[8] L. Shu, X. Wei, L. Jin, Y. Li, H. Wang, and X. Yao, Appl. Phys. Lett. 102, 152904 (2013).

[9] J. Narvaez and G. Catalan, Appl. Phys. Lett. 104, 162903 (2014).

[10] H. Lu, C. W. Bark, D. E. D. L. Ojos, J. Alcala, C. B. Eom, G. Catalan, and A. Gruverman, Science 335, 59
(2012).

[11] R. Maranganti, N. D. Sharma, and P. Sharma, Phys. Rev. B 74, 014110 (2006).

[12] N. D. Sharma, R. Maranganti, and P. Sharma, J. Mech. Phys. Solids 55, 2328 (2007).

[13] M. Gharbi, Z. H. Sun, P. Sharma, and K. White, Appl. Phys. Lett. 95, 142901 (2009).

[14] C. R. Robinson, K. W. White, and P. Sharma, Appl. Phys. Lett. 101, 122901 (2012).

[15] H. Zhou, Y. Pei, F. Li, H. Luo, and D. Fang, Appl. Phys. Lett. 104, 061904 (2014).

[16] W. Huang, X. Yan, S. R. Kwon, S. Zhang, F. G. Yuan, and X. Jiang, Appl. Phys. Lett. 101, 252903 (2012).

[17] A. Abdollahi, C. Peco, D. Millán, M. Arroyo, and I. Arias, J. Appl. Phys. 116, 093502 (2014).

[18] C. T. Sun and S. B. Park, Ferroelectrics 248, 79 (2000).

[19] G. A. Schneider, Annu. Rev. Mater. Res. 37, 491 (2007).

[20] A. Abdollahi and I. Arias, Acta Mater. 59, 4733 (2011).

[21] A. Abdollahi and I. Arias, Model. Simul. Mater. Sci. Eng. 19, 074010 (2011).

[22] Y. Jiang, D. Fang, and F. Li, Appl. Phys. Lett. 90 (2007).

[23] Y. Li and F. Li, Scripta Mater. 67, 601 (2012).

[24] I. Naumov, A. M. Bratkovsky, and V. Ranjan, Phys. Rev. Lett. 102, 217601 (2009).

[25] A. Abdollahi and I. Arias, J. Mech. Phys. Solids 60, 2100 (2012).

[26] G. Catalan, L. J. Sinnamon, and J. M. Gregg, J. Phys. Cond. Matt. 16, 2253 (2004).

[27] N. D. Sharma, C. M. Landis, and P. Sharma, J. Appl. Phys. 108, 24304 (2010).

[28] N. D. Sharma, C. Landis, and P. Sharma, J. Appl. Phys. 111, 059901 (2012).

[29] A. E. Eliseev, A. N. Morozovska, M. D. Glinchuk, and R. Blinc, Phys. Rev. B 79, 165433 (2009).

[30] N. D. Sharma, C. Landis, and P. Sharma, Journal of Applied Physics 111, (2012).

[31] S. Mao and P. K. Purohit, J. Appl. Mech. 81, 081004 (2014).

[32] S. M. Kogan, Sov. Phys. Solid State 5, 2069 (1964).

[33] M. Arroyo and M. Ortiz, Int. J. Numer. Meth. Eng. 65, 2167 (2006).

[34] R. M. McMeeking, Eng. Fract. Mech. 64, 217 (1999).

[35] W. Y. Li, R. M. McMeeking, and C. M. Landis, Eur. J. Mech. A-Solids 27, 285 (2008).

[36] Z. Li, S. K. Chan, M. H. Grimsditch, and E. S. Zouboulis, J. Appl. Phys. 70, 7327 (1991).

[37] R. Maranganti and P. Sharma, Phys. Rev. B 80, 054109 (2009).

[38] I. Ponomareva, A. K. Tagantsev, and L. Bellaiche, Phys. Rev. B 85, 104101 (2012).

[39] W. J. Merz, Phys. Rev. 76, 1221 (1949).

[40] J. Narvaez, S. Sareminaeini, J. Hong, and G. Catalan, Manuscript in preparation (2015).

[41] A. K. Tagantsev and A. S. Yurkov, J. Appl. Phys. 112, 044103 (2012).

[42] M. Stengel, Phys. Rev. B 90, 201112 (2014).

[43] J. Junquera and P. Ghosez, Nature 422, 506 (2003).

[44] Q. Deng, L. Liu, and P. Sharma, J. Mech. Phys. Solids 62, 209 (2014).

[45] Q. Deng, L. Liu, and P. Sharma, Phys. Rev. E 90, 012603 (2014).

[46] A. Livne, E. Bouchbinder, and J. Fineberg, Phys. Rev. Lett. 101, 264301 (2008). 